\title{
Clinical Profile of Breast Cancer in a Rural Population of India and Role of Modified Radical Mastectomy
}

\author{
Harish Gopalrao Umrajkar ${ }^{1}$, Pradeepta Barai ${ }^{2}$ \\ ${ }^{1}$ Associate Professor, Department of Surgery, ${ }^{2}$ Senior Registrar, Department of Surgery, Dr. Shankarrao Chavan Government \\ Medical College and Hospital, Nanded, Maharashtra, India
}

Corresponding author: Pradeepta Barai, Resident Quarters, Sassoon General Hospital, Pune, Maharashtra, India

DOI: http://dx.doi.org/10.21276/ijcmsr.2019.4.4.39

How to cite this article: Harish Gopalrao Umrajkar, Pradeepta Barai.Clinical profile of breast cancer in a rural population of india and role of modified radical mastectomy. International Journal of Contemporary Medicine Surgery and Radiology. 2019;4(4):D163-D167.

\section{A B S T R A C T}

Introduction: Breast cancer is the leading cause of morbidity and mortality among all cancers in the world, including India. Advances in multimodal therapy and genetic profiling haven't yet percolated into rural healthcare system mainly due to lack of availability, affordability and awareness. Modified radical mastectomy (MRM) performed by general surgeons may be an attempt for curative management in the appropriately selected patients. This study was carried out to analyze the clinical profile of breast cancer patients in a government medical college hospital serving the rural population of India and elucidate the role of general surgeons in providing modified radical mastectomy as early and comprehensive treatment.

Material and methods: A total of 72 patients confirmed as breast cancer were enrolled in this study. Patients with locally advanced and metastatic disease were excluded from the study. The patients defined as operable were followed up for 2 years after MRM and adjuvant therapy in order to investigate for any evidence of recurrence or metastatic disease.

Results: This study shows the clinical profile of breast cancer patients in rural India as opposed to that seen in urban population of India or western countries. Lack of resources and financial constraints in this setting limits treatment option. it was found that timely treatment with MRM, and after completion of adjuvant therapy wherever indicated, there was no evidence of local recurrence or systemic disease within a follow up period of 2 years.

Conclusion: MRM performed by general surgeons having reasonable experience in surgical oncology, for appropriately selected patients in a resource-limited setting of rural India, is a viable treatment option for curative intent.

Keywords: Breast Cancer; Clinical Profile; Rural; India; Mastectomy; General Surgeons.

\section{INTRODUCTION}

Over the last few decades, the alarming rise in breast cancer incidence in India has proven itself to be a burgeoning health problem, to an extent that now it is one of the most common types of cancer among women. ${ }^{1,2,3,4}$ Although breast cancer demographics in western countries have not changed significantly over the years possibly due to access to better treatment facilities as well as increased awareness and implementation of screening programs, the same cannot be said in case of Asian countries and particularly India, where despite of programs such as NPCDCS (National Programme for prevention and control of Cancer, Diabetes, Cardiovascular diseases and Stroke), there is an annual increase in incidence of breast cancer in most cancer registries in India. The greatest increase in incidences of breast cancer has been in Asian countries, where the clinical profile of the disease is different from that in western countries. ${ }^{5}$ A study conducted by Murthy NS Et al showed the time trends in incidence of breast cancer among various age group in India. According to it, the annual increase in breast cancer incidence in India ranges from $1.4 \%$ to about $6 \%$ in the oldest age group. ${ }^{6}$ In India, Breast cancer accounts for $25 \%$ of all female cancers $^{7,8}$, which amounts to approximately 75000 new cases every year. ${ }^{9}$ A majority of these patients present in a locally advanced stage $\mathrm{e}^{9,10}$, where a multidisciplinary approach to treatment becomes crucial.

As per the ICMR-PBCR data, breast cancer is the commonest cancer among women in most population-based urban registries where it constitutes $>30 \%$ of all cancers in females. ${ }^{11}$

The management of breast cancer becomes more challenging in the rural population as compared to urban population, where factors like access to resources like radiotherapy and mammography is limited, compliance to therapy is questionable and, high costs etc. prove to be a hinderance in providing comprehensive patient care to these patients.

Oncoplastic surgery options like lumpectomy and breast conservation surgery (BCS) is also underutilized for treatment of these patients due to advanced stage at presentation and limited access to resources in the rural healthcare system, which makes them a less feasible treatment strategy. All these factors entail a disproportionate mortality of breast cancer patients in rural population viz a viz urban population. This ultimately outlines the need for surgical treatment available at these places. 
In such a scenario, general surgeons having reasonable experience in surgical oncology may play a crucial role in providing comprehensive management of the disease in this population, where they can offer modified radical mastectomy (MRM) in the appropriately selected patients. The present study was conducted in a tertiary care institute (medical college and hospital) to understand the clinical profile of breast cancer in a rural population and further, to elucidate the feasibility of MRM done by general surgeons with reasonable oncosurgery experience, as a primary modality of treatment in appropriately selected patients in a rural population.

\section{MATERIAL AND METHODS}

The present hospital-based longitudinal study was conducted at Dr. Shankarrao Chavan Government medical college and hospital, Nanded. After ethics committee approval, patients were recruited from the IPD of the surgery department. A written informed consent was taken from all the patients. During the study period i.e. January 2012 to December 2012, a total of 72 patients were confirmed as breast cancer on histopathology of trucut biopsy specimen or fine needle aspiration cytology, after ultrasonography of breast as primary modality for imaging instead of mammography due to non-availability.

All patients were diagnosed as infiltrating ductal carcinoma (IDC). Patients with locally advanced breast cancer (LABC) or metastatic disease were excluded from the purpose of study for post-surgical outcomes.

Patients were staged according to AJCC $8^{\text {th }}$ edition. ${ }^{12}$ Stage $\mathrm{Ia}$ and Ib, IIa and IIb were included in study for MRM. Although BCS is indicated in majority of early breast cancers, due to non-availability of mammography and radiotherapy facility, patients were given the option of MRM after counselling. Modified Patey's mastectomy was done in all patients undergoing MRM in the study. Complete axillary lymph node dissection (ALND) was done with excision of level I and level II nodes (inferior and posterior to pectoralis minor respectively), with monopolar and bipolar cautery being used as energy source. All the patients undergoing MRM were operated by consultant general surgeons. Patients who were excluded from the study did not undergo MRM and were referred to higher centers for multimodal therapy. All ER/PR positive patients were given hormonal therapy with tamoxifen. All patients found to have axillary lymph nodal metastasis on HPE were given Postoperative chemoradiation at higher centers. Radiotherapy was offered to selected cases of high-grade tumor and with axillary spread. All patients tolerated the adjuvant therapy regimen well.

There were no clear guidelines for follow up in breast cancer patients in a rural setup, thus after completion of chemotherapy and radiotherapy, patients were followed up every 6 months with history and clinical examination, along with laboratory investigations (liver function test with alkaline phosphatase, complete blood count), chest X-ray, ultrasonography of abdomen and pelvis, and High resolution CT scan (in selected cases) of chest in selected cases after 2 years
Patients were followed for 2-year period till December 2014. On follow up, local, regional, and systemic recurrence along with post-operative outcomes were investigated.

\section{RESULTS}

In this study it was found that, median age of patients was 57 years. Minimum age of patient was 32 and maximum age was 82 years. Out of 72 patients in this study, 14 patients (19\%) were of stage I, 40 patients (55\%) were of stage II, 13 patients (18\%) were of stage III and 5 patients (7\%) were of stage IV [table 1]. Metastasis to liver was most common, seen in 4 patients (5.5\%) and, 1 patient (1.3\%) had metastasis to lungs. 3 patients (4.1\%) had family history of breast cancer.

$75 \%$ of the patients studied were found to be operable and thus had MRM done. The remaining patients of Stage III and IV were excluded from the study for MRM and were counselled and sent for multimodal therapy at a higher center. Regarding comorbidities among 72 patients who were studied, 6 patients (8\%) had history of fibroadenoma, 3 patients (4\%) had ischemic heart disease, 3 patients (4\%) had diabetes mellitus, 3 patients (4\%) had hypertension, 2 patients $(2.7 \%)$ had history of tuberculosis and 1 patient $(1.3 \%)$ had rheumatic heart disease.

31 patients (43\%) were premenopausal and 41 patients (57\%) were post-menopausal. [table 2].

Among the 31 patients (43\%) who were pre-menopausal, 12 patients (38\%) had stage I and II disease each, 5 patients (16\%) had stage III disease and 2 patients (5.4\%) had stage IV disease [table 3].

Of the 41 post-menopausal patients (56\%), 2 patients (4.8\%) had stage I disease, 28 patients $(68 \%)$ had Stage II disease, $8(19.5 \%)$ patients had stage III disease and, 3 Patients (7\%) had stage IV disease [table 4].

Regarding parity of patients, 9 patients (12\%) were nulliparous, with median age of 45 years, and 63 patients (87\%) were multiparous with median age of 57 years [Table 5]. Among these patients, 46 patients (63\%) had their menarche documented, with median age at menarche at 10.5 years. 35 patients (76\%) out of these documented 46 patients, had menarche before 12 years of age. This shows the correlation of earlier exposure of estrogen to propensity of suffering from breast cancer.

It was found that 22 patients (30\%) were having age less than 40 years, conferring a rising trend in breast cancer among patients who are relatively younger. 54 patients (75\%) underwent MRM. Of these 54 patients, 18 patients (33\%) were of stage III and IV were referred to higher center for multimodal therapy. Pathological findings such as receptor status, tumor grade, axillary lymphatic spread was inferred from those patients of stage I and II, who had MRM done. 30 patients $(55 \%)$ were receptor positive. 15 patients (27\%) had higher grade malignancy. 35 patients (64\%) had lymphovascular invasion on axillary nodal study given by histopathology report.

Median length of hospital stay was 12 days. There were 3 patients $(5.5 \%)$ with post-operative complication of seroma. 5 patients $(10 \%)$ had post-operative arm numbness. There were no cases of post op lymphedema or restricted shoulder movement. [table 6]. 


\begin{tabular}{|l|c|}
\hline Stage (overall) & No of patients \\
\hline I & 14 \\
\hline II & 40 \\
\hline III & 13 \\
\hline IV & 5 \\
\hline \multicolumn{2}{|c|}{ Table-1: Staging of patients } \\
\hline
\end{tabular}

\begin{tabular}{|l|c|}
\hline Menstrual status & No of patients \\
\hline Pre-menopausal & $31 / 72$ \\
\hline Post-menopausal & $41 / 72$ \\
\hline \multicolumn{2}{|c|}{ Table-2: Menstrual status of patients } \\
\hline
\end{tabular}

\begin{tabular}{|l|c|}
\hline Stage (among pre-menopausal) & No of patients \\
\hline I & 12 \\
\hline II & 12 \\
\hline III & 5 \\
\hline IV & 2 \\
\hline \multicolumn{2}{|c|}{ Table-3: Staging among pre-menopausal patients } \\
\hline
\end{tabular}

\begin{tabular}{|l|c|}
\hline Stage (among post-menopausal) & No of patients \\
\hline I & 2 \\
\hline II & 28 \\
\hline III & 3 \\
\hline IV & 3 \\
\hline \multicolumn{2}{|c|}{ Table-4: Staging among post-menopausal patients } \\
\hline
\end{tabular}

\begin{tabular}{|l|c|}
\hline Parity & No of patients \\
\hline null & 9 \\
\hline multiparous & 63 \\
\hline \multicolumn{2}{|c|}{ Table-5: Parity of patients } \\
\hline
\end{tabular}

\begin{tabular}{|l|c|}
\hline Post-operative complications & No of patients \\
\hline seroma & $3 / 54$ \\
\hline Arm numbness & $5 / 54$ \\
\hline \multicolumn{2}{|c|}{ Table-6: Post-operative complications } \\
\hline
\end{tabular}

2 years after surgery, no patient had locoregional recurrence or metastatic disease.

\section{DISCUSSION}

Breast cancer has emerged as the most common site of cancers occurring in women worldwide, including India.

The prevalence and incidence of the disease has been steadily increasing over the last few decades, outnumbering cervical cancer and lung cancer in most women population throughout the world. A study done by Nandkumar et al also highlights this, showing that newly diagnosed cases for breast cancer have overtaken cancer cervix as leading site of cancer in most Population Based Registries in India. ${ }^{13}$

The greatest increase in incidences has been in Asian countries, where the clinical profile of the disease is different from that in western countries. ${ }^{5}$

In Asia, breast cancer incidence peaks among women in their forties ${ }^{5}$, whereas in the United States and Europe, it peaks among women in their sixties. This variation in incidence is due to multiple factors, including geographic variation, racial/ ethnic background, genetic variation, lifestyle, environmental factors, socioeconomic status, the prevalence of known risk factors, utilization of screening mammography, stage of disease at diagnosis, and the availability of appropriate care. ${ }^{13}$ The epidemiology of breast cancer is shaped by geographical variation and availability and affordability of treatment options, such that although incidence rates in India are not as high compared to western countries, the mortality rate is at par with them. ${ }^{14,15}$

This may be due to higher incidence of the disease being offset by better treatment facilities in western countries, where access to advances in care such as gene profiling (GeneMap), neoadjuvant therapy, targeted therapy and hormonal therapy, or treatment modalities like oncoplastic surgery are not limited by availability or costs.

Early age at menarche $(<12$ years) is considered as a risk factor for developing breast cancer. In this study it was found that 35 of the 46 patients $(76 \%)$ in whom age at menarche was known, had menarche at less than 12 years of age.

It is also a matter of concern that breast cancer is increasingly being diagnosed in younger women in India than it was before. ${ }^{7,11}$ Increasing use of oral hormonal contraceptives, late menarche, radiation exposures, smoking all have been attributed to the breast cancer in younger population.

In addition to this, genetic tendency in Asian women to develop the disease, and environmental factors hitherto unknown may also be a cause of shifting trends towards a younger 9population at diagnosis. This study shows that about one in every three patients (30\%) is of less than 40 years of age.

It has been shown in this study that the disease peaks at 40 -50 years of age. In this study, it has been shown that mean age of presentation was 51 years and median age was 57 years. It has been shown that changing life styles in metropolitan cities like delayed marriage, late age at first child birth, lower parity and higher socio-economic status, may constitute some of the probable causes for higher incidences of breast cancer in urban as opposed to rural areas. ${ }^{16}$

Differences in the prevalence of central adiposity and age at first full-term pregnancy between rural and urban women from India may explain some differences in breast cancer incidence rates between these two populations. ${ }^{17}$

This was also indicated in the National Family Health Survey India (NFHS-3): 2005-06, where it was shown that differences in the incidence of breast cancer in rural as compared to urban region may be due to differences in reproductive factors such as a woman in urban India has an average of 2.1 children in her lifetime when compared to 3.0 children in rural India. Furthermore, the obesity pattern is lower $(8.6 \%)$ in rural areas as compared to the urban areas (28.9\%) of women in India. ${ }^{18}$

However, the burden of disease in rural India is clearly shown by a higher mortality to incidence ratio in rural population as compared to urban population (0.66 vs 0.08$))^{7}$ This also shows that benefits of lower incidence of breast cancer in rural population of India may have been counterbalanced by factors like advanced stage of presentation, lack of awareness and education which hampers health seeking behaviors, lack 
of screening programs and, lack of accessibility to treatment facilities for early diagnosis and treatment, leading to a mortality rate that is similar to urban areas. ${ }^{19,20}$

A study conducted by Dixit $\mathrm{R}$ et al showed that between the study period of 2002-03, mortality due to breast cancer among women was third most common (10.6\%) after cervical cancer $(17.1 \%)$ and stomach cancer $(14.1 \%) .{ }^{21}$

In face of the current scenario, role of general surgeons becomes even more important, where they can offer MRM as a part of comprehensive care in the appropriately selected and counselled patients. Although BCS is the standard of care in early breast cancer, issues like compliance to postoperative protocols regarding radiotherapy, need of highquality imaging like mammography and MRI for tumor localization, reliable histopathology for intraoperative cytology or frozen sections, possibility of re-excision surgery etc. makes BCS as a less feasible option in the management of these patients in the rural population.

In this study, 54 of the 72 patients that were enrolled were found operable and treated with modified radical mastectomy by general surgeons.

$55 \%$ of these patients were either ER or PR positive. This is comparable to the ER/PR positivity rate found in a study done by Nene BM et al which showed 29\% ER positivity and $26 \%$ PR positivity. 22 27\% of the patients had high grade malignancy. These findings show the need of hormonal therapy and radiotherapy, in the treatment plan of these patients. $64 \%$ of these patients had lymphovascular invasion on axillary nodal study. This shows that local metastasis occurs early in the course of this disease.

Besides a few established factors such as body mass index, the use of electrocautery for dissection, early drain removal, low vacuum drains, obliteration of dead space, and delayed shoulder physiotherapy, most of the hypothesized causes of seroma formation were not consistently demonstrated. ${ }^{23}$

In this study, it was found that seroma formation after MRM occurred in 3 patients. Deo SV et al studied the difference in seroma formation after MRM using harmonic scalpel or electrocautery and it was found that difference in rate of seroma formation was not significant between the two modalities. (16\% vs $22 \%) .{ }^{24}$

Complications of Post-operative arm morbidity caused by arm numbness or lymphedema was also studied. $10 \%$ of the patients who were treated by MRM had post-operative arm numbness. Roses DF et al studied the complications of level I and level II ALND and found that $76.5 \%$ patients had numbness or paresthesias of the medial arm and/or axilla after surgery; in $82 \%$ of these, the problem had lessened or had resolved on follow-up assessment. ${ }^{24}$ Maunsell E et al studied arm-related post -operative complications and found that $58 \%$ of patients had complains of some degree of arm numbness. ${ }^{25}$

None of the patients reported any symptoms of lymphedema in the follow-up period.

After a follow up period of 2 years, no patient was found to have any signs of local recurrence or distant metastasis. This shows that MRM done by general surgeons with reasonable experience in oncosurgical principles, is appropriate and feasible in the treatment of breast cancer patients in rural population of India, where access of and compliance to ancillary modality of treatment and investigations like radiotherapy, chemotherapy, mammography, MRI etc. is limited by affordability, lack of awareness and lack of education. Thus, MRM may be an effective primary modality of treatment in these patients which also reduces mortality in a patient population where it is disproportionately high.

Thus, Non-availability of mammographic evaluation and Radiotherapy facilities due to physical or economic barriers, coupled with poor socio-economic status, compliance to therapy, early propensity of high-grade tumor to metastasize and cause lymphovascular invasion, all these factors necessitate need for proper surgical management of these patients in rural setups. Thus, MRM gives effective treatment of breast cancer for overall survival in these conditions.

\section{CONCLUSION}

After carrying out this study, we found that that epidemiology and presentation of breast cancer in rural population of India poses serious challenges such as, rising trend of breast cancer in relatively younger patients, advanced stage of presentation, and limited access and affordability for standard of care in this population. Further, with very little awareness and lack of screening programs coupled with resource limited nature of rural healthcare system, management and prognosis of these patients gets hampered. Considering that the degree of post-operative complication such as seroma formation, arm numbness etc. is at par with studies conducted previously, along with absence of recurrence of the disease in a period of 2 years, it may be concluded that general surgeons who are adept in oncologic principles, have an important role to play in this setting, where they can offer MRM to appropriately selected group of patients in order to attempt a comprehensive curative management.

However further studies and longer follow-up period are needed, in order to ascertain the efficacy of treatment provided by general surgeons in this patient population, where treatment for curative intent seems to have greater benefits than risks.

\section{REFERENCES}

1. Dumitrescu RG, Cotarla I. Understanding breast cancer risk-where do we stand in 2005? J Cell Mol Med 2005;9(1):208-21.

2. Chandra AB. Problems and prospects of cancer of the breast in India. J Indian Med Assoc 1979;72(6):43-5.

3. Jemal A, Bray F, Center MM, et al. Global cancer statistics. CA Cancer J Clin 2011;61(3):69-90.

4. Stewart BW, Wild CJ. WHO. World Cancer Report 2014. 2014; International Agency for Research on Cancer, Lyon, France:630.

5. Green M, Raina V. Epidemiology, screening and diagnosis of breast cancer in the Asia-Pacific region: current perspectives and important considerations. Asia Pacific J Clin Oncol 208;4(Suppl 3):S5-S13

6. Murthy NS, Chaudhry K, Nadayil D, Agarwal UK, Saxena S. Changing trends in incidence of breast cancer: Indian scenario. Indian J Cancer. 2009;46(1):73-4.

7. Malvia S, Bagadi SA, Dubey US, et al. Epidemiology of breast cancer in Indian women. Asia Pac J Clin Oncol 
2017;13(3):289-95.

8. Programme NCR. Three year report of populationbased cancer registries: 2009-2011. In: Indian Council of Medical Research, NCDIR-NCRP (ICMR) Bangalore; 2013.

9. Chopra R. The Indian Scene. Journal of Clinical Oncology 2001;19(1):S106-11.

10. Agarwal G, Pradeep PV, Aggarwal V, Yip CH, Cheung PS. Spectrum of breast cancer in Asian women. World J Surg 2007; 31(4):1031-40.

11. National Cancer Registry Programme. Consolidated report of the population-based cancer registries 19901996. New Delhi: Indian Council of Medical Research; 2001.

12. Armando E. Giuliano, Stephen B. Edge, Gabriel N. Hortobagyi. Eighth Edition of the AJCC Cancer Staging Manual: Breast Cancer. Annals of Surgical Oncology; 2018;25(7):1783-1785.

13. Nandakumar, et al. Three-year population report of population-based cancer registries 2009-11. Bangalore: National Centre for Disease Informatics and ResearchNational Cancer Registry Programme (ICMR); 2013

14. Hortobagyi GN, de la Garza Salazar J, Pritchard $\mathrm{K}$ et al. The global breast cancer burden: variations in epidemiology and survival. Clin Breast Cancer 2005;6(2):391-401

15. Gupta A, Shridhar K, Dhillon PK. A review of breast cancer awareness among women in India: cancer literate or awareness deficit? Eur J Cancer 2015; 51(3): 205866.

16. Ferlay J, Soerjomataram I, Ervik M, Dikshit R, Eser S, Mathers C, et al. Estimated Cancer Incidence, Mortality and Prevalance Worldwide in 2012. 2012. v1.0 (IARC CancerBase No. 11).

17. Manoharan N, Nair O, Shukla NK, Rath GK. Descriptive Epidemiology of Female Breast Cancer in Delhi, India. Asian Pac J Cancer Prev. 2017;18(4):10151018.

18. Nagrani R, Mhatre S, Boffetta P, Rajaraman P, Badwe R, Gupta S, Romieu I, Parmar V, Dikshit R. Understanding rural-urban differences in risk factors for breast cancer in an Indian population. Cancer Causes Control. 2016;27(2):199-208.

19. National Family Health Survey India (NFHS-3): 2005-06. Maharashtra, India: International Institute for Population Sciences (IIPS); 2008

20. Agarwal $G^{1}$, Ramakant $P$ Breast Cancer Care in India: The Current Scenario and the Challenges for the Future. Breast Care (Basel). 2008;3(1):21-27.

21. Dikshit R, Gupta PC, Ramasundara hettige C, Gajalakshmi V, Aleksandrowicz L, Badwe R, et al. Cancer mortality in India: A nationally representative survey. Lancet 2012;379(5):1807-16.

22. Nene BM, Selmouni F, Lokhande M, Hingmire SJ, Muwonge R, Jayant K, Sankaranarayanan R. Patterns of Care of Breast Cancer Patients in a Rural Cancer Center in Western India. Indian J Surg Oncol. 2018;9(3):374380.

23. Deo SV, Shukla NK, Asthana S, Niranjan B, Srinivas G. A comparative study of modified radical mastectomy using harmonic scalpel and electrocautery. Singapore
Med J. 2002;43:226-8.

24. Srivastava V, Basu S, Shukla VK. Seroma formation after breast cancer surgery: what we have learned in the last two decades. J Breast Cancer. 2012;15(4):373-80.

25. Maunsell $\mathrm{E}^{1}$, Brisson J, Deschênes L. Arm problems and psychological distress after surgery for breast cancer. Can J Surg. 1993;36(4):315-20.

\section{Source of Support: Nil; Conflict of Interest: None}

Submitted: 14-11-2019; Accepted: 30-11-2019; Published online: 25-12-2019 